\title{
The effect of cirrus clouds on microwave limb radiances
}

\author{
C. Emde ${ }^{a, *}$, S.A. Buehler ${ }^{a}$, P. Eriksson ${ }^{\text {b }}$ T.R. Sreerekha ${ }^{a}$ \\ ${ }^{a}$ Institute of Environmental Physics, University of Bremen, Otto-Hahn-Allee 1, D-28359 Bremen, Germany \\ ${ }^{\mathrm{b}}$ Department of Radio and Space Science, Chalmers University of Technology, SE-41296 Göteborg, Sweden
}

Received 1 August 2003; received in revised form 15 January 2004; accepted 31 March 2004

\begin{abstract}
This study presents and analyses the first simulations of microwave limb radiances with clouds. They are computed using the 1D unpolarized version of the Atmospheric Radiative Transfer System (ARTS). The study is meant to set a theoretical foundation for using microwave limb measurements for cloud monitoring. Information about clouds is required for the validation of climate models.

Limb spectra are generated for the frequency bands of the Millimeter wave Acquisitions for Stratosphere/Troposphere Exchange Research (MASTER) instrument. For these simulations, the radiative transfer equation is solved using the Discrete Ordinate ITerative (DOIT) method, which is briefly described. Single scattering properties for the cloud particles are calculated using the T-matrix method.

The impact of various cloud parameters is investigated. Simulated brightness temperatures most strongly depend on particle size, ice mass content and cloud altitude. The impact of particle shape is much smaller, but still significant. Increasing the ice mass content has a similar effect as increasing the particle size; this complicates the prediction of the impact of clouds on microwave radiances without exact knowledge of these cloud parameters.
\end{abstract}

(C) 2004 Elsevier B.V. All rights reserved.

Keywords: Radiative transfer; Microwave remote sensing; Scattering; Cirrus clouds; Limb observations

\section{Introduction}

To improve existing climate models, it is very important to extend the knowledge about cirrus cloud parameters, as such clouds cover more than $20 \%$ of the globe (Wang et al.,

* Corresponding author.

E-mail address: emde@uni-bremen.de (C. Emde).

URL: http://www.sat.uni-bremen.de. 
1996) and play an important role in the earth's radiation budget (Arking, 1991). Depending on cloud altitude and microphysical properties, clouds can either cause warming or cooling at the earth's surface. So far, clouds are not well treated in global climate models (GCM) because of uncertainties concerning the properties of cirrus clouds and complex interaction between radiation, microphysics and dynamics in these clouds. Moreover, it is essential to consider clouds for the evaluation of limb measurements of trace gases in the upper troposphere. Measurements contaminated by clouds have to be detected and corrected appropriately (e.g., Read et al., 2001).

In particular, the effective radius $R_{\text {eff }}$ of cloud particles is important to investigate the radiative properties of clouds. For a given frequency, $R_{\text {eff }}$ largely determines the relation between ice mass content (IMC) and cloud optical thickness (Evans et al., 1998). Parameterizations of $R_{\text {eff }}$ have been retrieved from combined lidar and radar reflectivity (Donovan, 2003) or from observations made in situ using aircraft mounted instruments (e.g., Kinne et al., 1997). The submillimeter-wave cloud ice radiometer (SWCIR) to fly on an aircraft has been developed to retrieve upper tropospheric and IMC and $R_{\text {eff }}$ (Evans et al., 2002).

Satellite remote sensing techniques in the thermal infrared can only be applied for thin cirrus clouds consisting of small ice particles as saturation is reached for moderate optical depths (Stubenrauch et al., 1999). Only ice particle properties of the uppermost cloud layers can be measured. Disadvantages of visible and near-infrared solar reflection methods include that they cannot distinguish between ice and liquid water clouds and they cannot measure low optical depth clouds over brighter land surfaces.

There are several studies about the sensitivity of cirrus clouds on microwave nadir radiances (e.g. Evans et al., 1998; Skofronik-Jackson et al., 2002). They show that the brightness temperature depression depends strongly on particle size and IMC. This is the first study of that kind for microwave limb radiances. Passive nadir-viewing techniques cannot sufficiently resolve the vertical distribution of IMC. Microwave limb techniques can provide higher resolutions than nadir techniques for the same frequencies. A technique for cloud detection and IMC retrieval for microwave limb measurements is described in Wu et al. (submitted for publication). The algorithm is applied to the Upper Atmospheric Research Satellite Microwave Limb Sounder (UARS MLS) 203-GHz radiance measurements. In this study, the impact of clouds on microwave limb radiance measurements of the planned Millimeter Wave Acquisitions for Stratosphere/Troposphere Exchange Research (MASTER) (Buehler, 1999) instrument is investigated.

A new version of the Atmospheric Radiative Transfer Simulator (ARTS) is used for the simulations. The first version of ARTS is a one-dimensional (1D) unpolarized model, which is sufficiently fast for operational use but does not include scattering (Buehler et al., in press). The new version is currently developed in order to simulate the influence of multiple scattering on measured radiances, polarization effects emerging from ice particle scattering and, in addition, three-dimensional (3D) effects resulting from horizontal inhomogeneities in the atmosphere. It can be applied in the microwave and in the infrared. ARTS has been compared to a forward model developed at the Rutherford Appleton Laboratory (RAL) (Kerridge et al., 2003) and to the single scattering forward model KOPRA developed for the Michelson interferometer for passive atmospheric sounding (MIPAS) (Hoepfner and Emde, in press). ARTS and 
the RAL forward model show an excellent agreement (less then $1 \mathrm{~K}$ difference in simulated brightness temperatures for most cloud cases), and ARTS and KOPRA agree well in the single scattering regime. For this study, we have used the 1D unpolarized version of ARTS.

In Section 2, the radiative transfer equation, which describes scattering, extinction and thermal emission in the atmosphere, is introduced. In Section 3, we will briefly describe our solution method, which is called Discrete Ordinate Iterative (DOIT) method. In Section 4, the setup for the model simulations is defined. We need general definitions concerning the sensor (altitude and viewing direction) and the atmosphere (geometry and composition). Furthermore, different cloud cases are defined. For calculating single scattering properties, we have used the T-matrix method. The numerical setup, which is essential for the accuracy of the results, also has to be specified. In Section 6, the results are presented. The importance of different quantities relevant in microwave radiative transfer through clouds is investigated. We concentrate on the impact of particle size, ice mass content (IMC), cloud height and frequency region. Particle shape is only a minor issue in this study. Spectra for various cloud types are presented and discussed.

\section{Radiative transfer equation}

The radiative transfer equation (RTE) for a medium with thermal emission comprising sparsely and randomly distributed arbitrarily oriented particles is according to Mishchenko et al. (2002) and Sreerekha et al. (2002).

$$
\frac{\mathrm{d} \boldsymbol{I}(\mathbf{n}, v)}{\mathrm{d} s}=-\langle\mathbf{K}(\mathbf{n}, v)\rangle \boldsymbol{I}(\mathbf{n}, v)+\langle\boldsymbol{a}(\mathbf{n}, v)\rangle B(v)+\int_{4 \pi} \mathrm{d} \mathbf{n}^{\prime}\left\langle\mathbf{Z}\left(\mathbf{n}, \mathbf{n}^{\prime}, v\right)\left(n^{\prime}, v\right)\right.
$$

where $\boldsymbol{I}$ is the Stokes vector, $\langle\mathbf{K}\rangle$ the ensemble-averaged extinction matrix, $\langle\boldsymbol{a}\rangle$ the ensemble-averaged absorption vector, $B$ the Planck function and $\langle\mathbf{Z}\rangle$ the ensembleaveraged phase matrix. Furthermore, $v$ is the frequency of the radiation, $\mathrm{d} s$ a pathlength-element of the propagation path and $\mathbf{n}$ the propagation direction. Eq. (1) is valid for monochromatic radiative transfer. We can use this equation for simulating microwave radiative transfer through the atmosphere, as the scattering events do not change the frequency of the radiation.

The four-component Stokes vector $\boldsymbol{I}=(I, Q, U, V)$ fully describes the radiation and it can directly be associated with the measurements carried out by a radiometer used for remote sensing. The first component $I$ is the intensity of the radiation, which is the only component studied in this paper. The other components are connected to the polarization state of the radiation. $Q$ is the difference between the intensities of the horizontally polarized and the vertically polarized part of the radiation. $U$ is also related to linear polarization and $V$ describes the circular polarization (Bohren and Huffman, 1998). 
Eq. (1) is an inhomogeneous differential equation. The three terms on the right-hand side of the equation describe physical processes in radiative transfer. The first term corresponds to the total extinction of the radiation along the path-length element $\mathrm{d} s$. Total extinction includes absorption by gases and particles in the atmosphere and also the amount of radiation scattered away from the propagation direction $\mathbf{n}$ into other directions $\mathbf{n}^{\prime}$. The second term, which is a radiation source term, describes the thermal emission. It is given by the product of the total absorption coefficient including gas and ensemble integrated particle absorption and the Planck function. Another source is radiation scattered from all directions $\mathbf{n}^{\prime}$ into the propagation direction $\mathbf{n}$. This contribution is expressed in the scattering integral given by an integration over all incoming directions $\mathbf{n}^{\prime}$. To evaluate this integral at a given point, we need the radiation field, i.e., the Stokes vectors for all incoming directions and the ensemble averaged phase matrix $\mathbf{Z}$. The phase matrix gives the information how the radiation is redistributed in space by a scattering event.

\section{Numerical solution method for the RTE}

We have developed the discrete ordinate iterative (DOIT) method to solve the RTE. The description presented in this section is an overview of the basic principle; details can be found in the ARTS user guide (Eriksson et al., 2003) and in Sreerekha et al. (2002) where the same approach has been applied and investigated in a plane parallel 1D radiative transfer model.

Since vertical and horizontal extent of cirrus clouds are finite, it is sufficient to solve the scattering problem in a restricted region. We denote the region where clouds possibly exist as the cloudbox. If there is a significant reflection from the earth's surface, the ice cloud and the surface can interact. In this case, the cloudbox is extended down to the ground which is then considered to be a scattering object.

The problem to solve is to find the radiation field inside the cloudbox. As it is not possible to find an analytical solution which would be a continuous representation of the radiation field, the cloudbox has to be discretized in the spatial domain and also in the angular domain of the propagation directions. The radiation field is a set of Stokes vectors which are calculated for each discrete point depending on five coordinates (pressure, latitude and longitude in the spatial domain and zenith and azimuth angle in the angular domain). The boundary condition is the incoming radiation field on the boundary of the cloudbox calculated by a clear-sky radiative transfer calculation.

In order to obtain a first guess $\boldsymbol{I}^{(0)}$ for the scattering integral field, the clear-sky field is interpolated on all discrete cloudbox points. The interpolated field is taken as a first guess to evaluate the scattering integral term. Putting the result of the integration into Eq. (1) leads to a simplified differential equation, which can be written as an iterative equation:

$$
\frac{\mathrm{d} \boldsymbol{I}^{(n)}(\mathbf{n}, v)}{\mathrm{d} s}(\mathbf{n}, v)=-\langle\mathbf{K}(\mathbf{n}, v)\rangle \boldsymbol{I}^{(n)}(\mathbf{n}, v)+\langle\boldsymbol{a}(\mathbf{n}, v)\rangle B(v)+\left\langle\boldsymbol{S}^{(n-1)}(\boldsymbol{n}, v)\right\rangle
$$


Here $\boldsymbol{I}^{(n)}$ is the $n$th iteration field and $\langle\boldsymbol{S}\rangle^{n-1}$ is the ensemble averaged scattering integral vector calculated using the $(n-1)$ th iteration field:

$$
\left\langle\boldsymbol{S}^{(n-1)}(\mathbf{n}, v)\right\rangle=\int_{4 \pi} \mathrm{d} \mathbf{n}^{\prime}\left\langle\mathbf{Z}\left(\mathbf{n}, \mathbf{n}^{\prime}, v\right)\right\rangle \boldsymbol{I}^{(n-1)}\left(\mathbf{n}^{\prime}, v\right)
$$

Assuming that the coefficients are constant between the discrete spatial grid points, Eq. (2) can be solved analytically using an exponential approach. In practice, we take averaged values for the coefficients. This approximation is valid if the discretization of the cloudbox is sufficiently fine.

The first iteration field $\boldsymbol{I}^{(1)}$, i.e., the field obtained by solving Eq. (2) for all discrete points, is compared to the first guess field $\boldsymbol{I}^{(0)}$. Assume that the first iteration field is closer to the solution field than the first guess field. In this case, it makes sense to repeat the procedure which is to calculate the scattering integral now using the first iteration field and then solving again Eq. (2). In this way, the second iteration field $\boldsymbol{I}^{(2)}$ is obtained.

On the assumption that the method converges, two successive iteration fields must be equal within a numerical accuracy limit. If this is achieved after the $n$th iteration, the $n$th iteration field is taken as solution of Eq. (1).

\section{Model setup for simulations of limb radiances}

\subsection{Atmosphere}

The simulations are performed in a 1D spherical model atmosphere. Of course, a 1D atmosphere is not a realistic environment for modeling clouds because clouds are horizontally strongly inhomogeneous. Each cloud included in a 1D model corresponds to full cloud coverage around the globe. Thus, the 1D calculations presented in this study can only be taken as an upper limit of scattering effects on the simulated radiances. Besides nitrogen and oxygen, the two major atmospheric gases, water vapour and ozone, are included. The concentrations are taken from FASCOD (Anderson et al., 1986) data for mid-latitudes in summer. Profiles for temperature, ozone and water vapor are shown in Fig. 1. Gas absorption is calculated based on the HITRAN (Rothman et al., 1998) molecular spectroscopic database using the ARTS model (first version). Refraction has been neglected in all calculations.

\subsection{Sensor setup}

The spectral ranges of bands B (293-306 GHz), C (317-326 GHz), D (342-349 GHz) and $\mathrm{E}(496-506 \mathrm{GHz})$ of the MASTER instrument are used for the simulations. The different scans correspond to tangent altitudes from 0 up to $12.5 \mathrm{~km}$, where $0 \mathrm{~km}$ altitude is the earth's surface and $12.5 \mathrm{~km}$ is a tangent altitude above the cloud. For simplicity, we assume the sensor to be ideal, which means that it measures exactly the intensity of the incoming radiation and is not subject to noise or other errors. This 

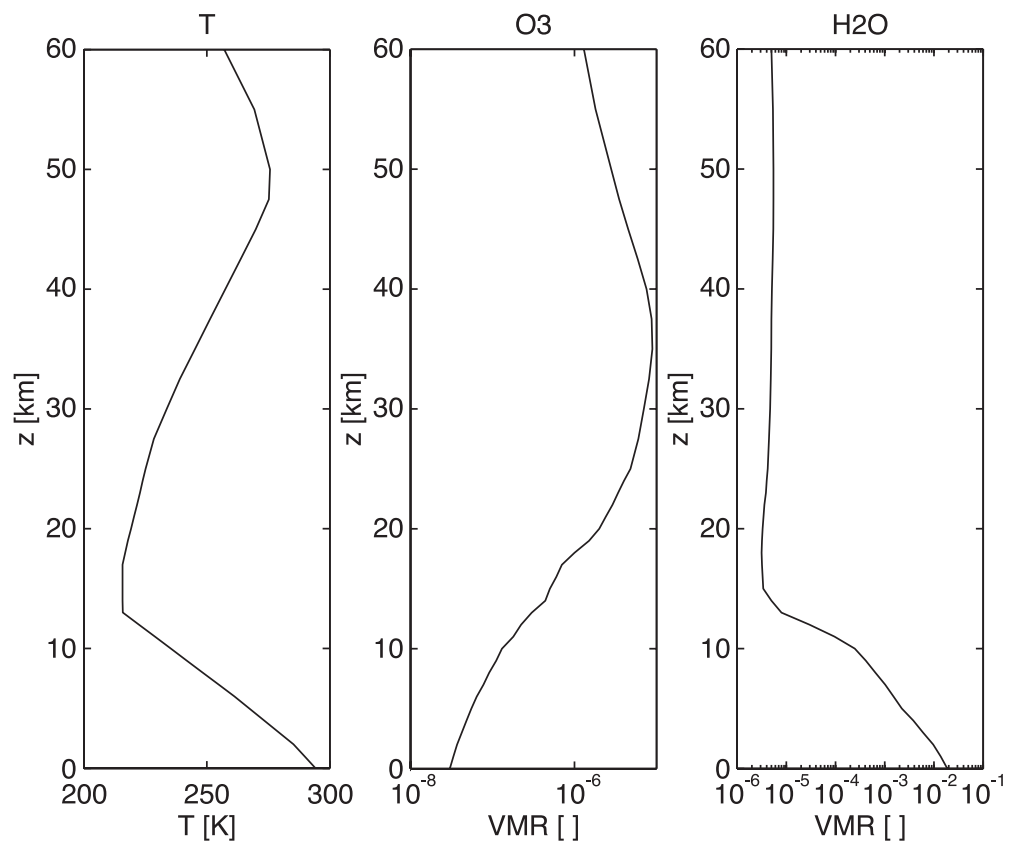

Fig. 1. Atmospheric profiles for temperature, ozone and water vapor (FASCOD).

implies that the signal neither depends on the antenna pattern nor on the polarization of the measured radiation. The scalar version of ARTS is applied. Here, only the first component of the Stokes vector is calculated; this means that polarization is neglected in the radiative transfer.

\subsection{Numerical setup}

For the accuracy of the results, the discretization, especially in the angular domain, is very important. For the calculation of the scattering integral [Eq. (3)], we have taken an equidistant zenith angle grid with an increment of $10^{\circ}$. This is not sufficient for the radiative transfer calculation [(Eq. (2)], as the radiation field is strongly inhomogeneous around $90^{\circ}$. For up-looking angles inside the cloudbox (below $90^{\circ}$ ), the amount of radiation is cosmic background, which corresponds to a very low brightness temperature of about $2.7 \mathrm{~K}$ and emission from gases above the cloud. This emission is only significant in the centers of the spectral lines. For down-looking angles (above $90^{\circ}$ ), the radiation emerges from the lower atmosphere and from the ground, resulting in brightness temperatures of more than $230 \mathrm{~K}$. We have taken a grid, which is optimized in such a way that it is able to represent the clear-sky radiation field with an accuracy of $0.1 \%$. For the iterated radiation fields, the inhomogeneity is smoothed out, so the accuracy is expected to be even better than $0.1 \%$. The radiation field and the scattered field are interpolated between the two zenith angle grids. The vertical grid is equidistant in altitude and the grid step-size is $0.5 \mathrm{~km}$. If a propagation path step, i.e., the distance 
of two successive intersection points of the line of sight (LOS) with the vertical grid, is longer than $1 \mathrm{~km}$, which occurs in limb geometry close to the tangent point, this step is divided in smaller $(<1 \mathrm{~km})$ equidistant steps.

\section{Definition of cloud scenarios}

\subsection{Size distribution and single scattering properties}

Cirrus clouds consist of size- and shape-distributed particle ensembles. For this study, we have used a gamma distribution to parameterize the particle sizes:

$$
n(r)=a r^{\alpha} \exp (-b r)
$$

The dimensionless parameter $\alpha$ describes the width of the distribution. In all calculations, $\alpha=1$ is assumed. The other two parameters can be linked to the effective radius and the ice mass content IMC, i.e., the mass of ice contained in a cubic meter of air, as follows:

$$
\begin{aligned}
& b=\frac{\alpha+3}{R_{\mathrm{eff}}} \\
& a=\frac{\mathrm{IMC}}{4 / 3 \pi \rho b^{-(\alpha+4)} \Gamma[\alpha+4]}
\end{aligned}
$$

where $\rho$ is the density of ice and $\Gamma$ is the gamma function.

Ensemble-averaged phase matrix, absorption vector and extinction matrix have been computed using the T-matrix code developed by Mishchenko and Travis (1998). The refractive index of ice, which is needed as an input to the T-matrix code, is computed using a code developed by Warren (1984). The T-matrix method is applicable for rotationally symmetric particles like spheres, cylinders or spheroids. We have chosen the T-matrix method instead of the simpler Mie theory for spherical particles because it is important to have the possibility to handle aspherical particles.

Fig. 2 shows extinction cross sections, scattering cross sections and absorption cross sections for gamma-distributed randomly oriented particle ensembles. The values are the averaged cross sections per particle. The particle shape is cylindrical with an aspect ratio of 0.5 . All coefficients are calculated using the T-matrix method. The scattering cross section increases nonlinearly with $R_{\text {eff }}$ in the frequency region below $1000 \mathrm{GHz}$. This feature appears also for spherical particles and can be explained, for example, by Mie theory (see, e.g., Bohren and Huffman, 1998; Mishchenko et al., 2002), which allows to calculate the scattering cross section as a function of frequency and particle size analytically. The peak observed in Fig. 2 corresponds to the first major maximum which belongs to the so-called "interference structure." The position of the peak is related to the particle size; for smaller particles, it shifts towards higher frequencies. The absolute value of the cross section at the maximum increases as well with increasing particle size. 


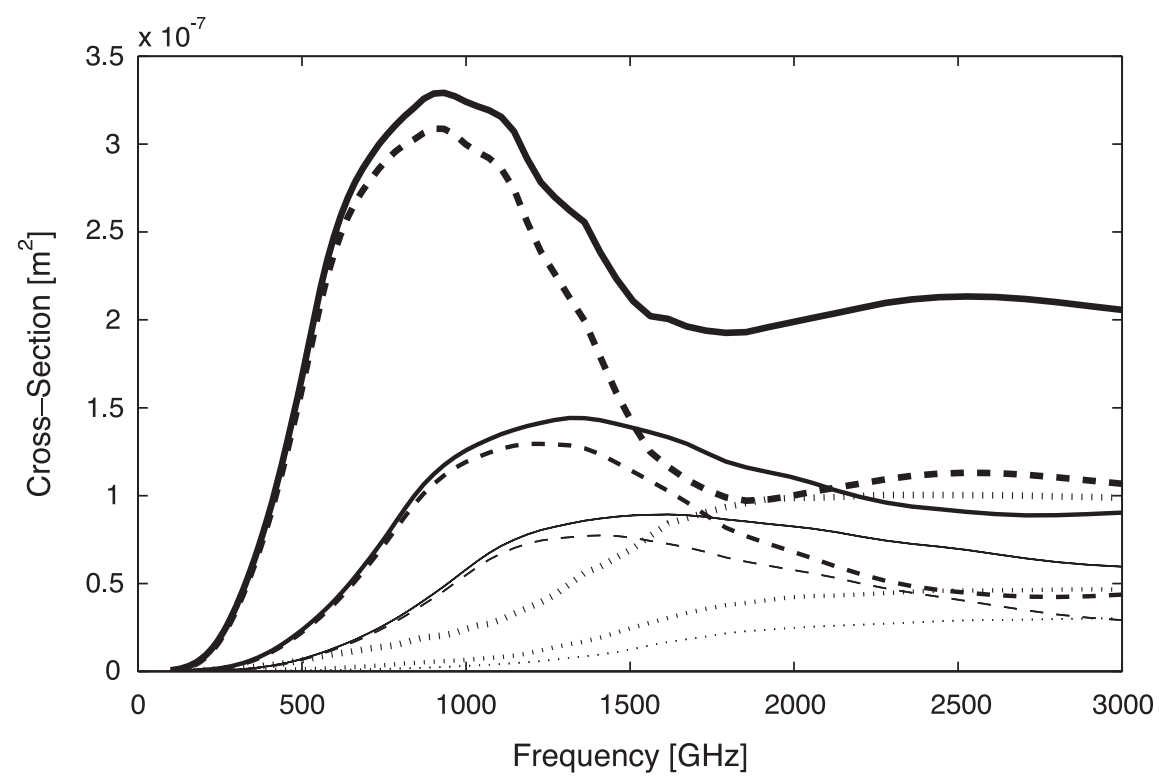

Fig. 2. Extinction cross section (solid lines), scattering cross section (dashed lines) and absorption cross section (dotted lines) for gamma-distributed randomly oriented particle ensembles of cylindrical particles with aspect ratio 0.5. Particle size distributions with $R_{\mathrm{eff}}=150 \mu \mathrm{m}$ (thick), $R_{\text {eff }}=100 \mu \mathrm{m}$ (medium) and $R_{\mathrm{eff}}=80 \mu \mathrm{m}$ (thin).

5.2. Setup for the investigation of the impact of different cloud properties on limb radiances

To study the impact of different cloud properties (IMC, particle size, particle shape and altitude and frequency), the test cases compiled in Table 1 have been defined. In all test cases except case (3), we assume that the cloud consists only of spherical particles. Band C is taken for all calculations except for case (5). As the scattering signal depends also on the

Table 1

Definition of five test cases to study the effect of cloud properties on limb radiances

\begin{tabular}{|c|c|c|c|c|c|}
\hline & $\operatorname{IMC}\left(\mathrm{g} / \mathrm{m}^{3}\right)$ & $R_{\text {eff }}(\mu \mathrm{m})$ & Cloud alt. (km) & Band & Aspect ratio \\
\hline 1 & $1.6 \times 10^{-3}$ & $\begin{array}{l}21.5,34.0,68.5 \\
85.5,128.5\end{array}$ & $10-12$ & $\mathrm{C}$ & - \\
\hline 2 & $1.6 \times 10^{-3}$ & 34.0 & $\begin{array}{l}6-8,8-10 \\
10-12\end{array}$ & $\mathrm{C}$ & - \\
\hline 3 & $1.6 \times 10^{-3}$ & 68.5 & $10-12$ & $\mathrm{C}$ & $\begin{array}{l}0.3,0.5,1.0, \\
2.0,4.0\end{array}$ \\
\hline 4 & $\begin{array}{l}4 \times 10^{-5} \\
1.6 \times 10^{-3} \\
8 \times 10^{-3} \\
0.016 \\
0.04\end{array}$ & $\begin{array}{l}21.5 \\
34.0 \\
68.5 \\
85.5 \\
128.5\end{array}$ & $10-12$ & $\mathrm{C}$ & - \\
\hline 5 & $1.6 \times 10^{-3}$ & 34.0 & $10-12$ & $\mathrm{~B}, \mathrm{C}, \mathrm{D}, \mathrm{E}$ & - \\
\hline
\end{tabular}


amount of gas absorption, it is interesting to study the effects in a band where we find frequency regions with high and others with low gas absorption (window regions). In this sense, band $\mathrm{C}$ is the best selection.

Cloud height $10-12 \mathrm{~km}$ means that the scattering properties are defined on all pressure grid points between 10 and $12 \mathrm{~km}$. The properties are linearly interpolated between the grid points; in this case, they are interpolated between 9.5 and $10 \mathrm{~km}$ and between 12 and $12.5 \mathrm{~km}$. Between 10 and $12 \mathrm{~km}$, the scattering properties are constant.

Table 1 includes the following scenarios:

(1) To investigate the impact of particle size, we have calculated spectra for the MASTER-C frequency band. The IMC is constant in all calculations $\left(1.6 \times 10^{-3} \mathrm{~g} /\right.$ $\mathrm{m}^{3}$ ) and the mean effective radius is varied from 21.5 to $128.5 \mu \mathrm{m}$.

(2) The influence of cloud altitude is also studied using band C. IMC and effective radius are constant, $1.6 \times 10^{-3} \mathrm{~g} / \mathrm{m}^{3}$ and $34.0 \mu \mathrm{m}$, respectively. Calculations are performed for three different cloud altitudes: $6-8,8-10$ and $10-12 \mathrm{~km}$.

(3) This is the only case where the cloud is assumed to consist of cylindrical particles. Spectra for five different aspect ratios in the range from 0.3 to 4.0 are calculated. The aspect ratio is the diameter of the cylinder divided by its length. Again, band $\mathrm{C}$ is used and IMC and effective radius are constant, $1.6 \times 10^{-3} \mathrm{~g} / \mathrm{m}^{3}$ and $68.5 \mu \mathrm{m}$, respectively.

(4) Measurements have shown that there is a correlation between particle size and ice mass content. We have picked out realistic scenarios according to a plot in Evans et al. (1998) which shows results from the FIRE (Kinne et al., 1997) campaign. The IMC is varied from $4 \times 10^{-5}$ to $0.04 \mathrm{~g} / \mathrm{m}^{3}$ and the effective radius from 21.5 to 128.5 $\mu \mathrm{m}$. As in the other cases, band $\mathrm{C}$ is taken and the cloud altitude is $10-12 \mathrm{~km}$.

(5) Calculations for different frequency bands, bands B, C, D and E, are performed for this case to see the impact of the same cloud in different frequency regions. IMC and effective radius are constant, $1.6 \times 10^{-3} \mathrm{~g} / \mathrm{m}^{3}$ and $34.0 \mu \mathrm{m}$, respectively.

\section{Results}

\subsection{Impact of particle size}

Simulations for varying particle sizes and constant IMC are shown in Fig. 3. The simulated radiances are presented in Rayleigh-Jeans brightness temperature (BT) units. On the left-hand side, we see the results at 8-km tangent altitude. The spectrum of the cloud consisting of the smallest particles $\left(R_{\text {eff }}=21.5 \mu \mathrm{m}\right)$ is almost identical to the clear-sky spectrum. All other clouds show a BT depression in the window regions. For a particle size of $34.0 \mu \mathrm{m}$, the brightness temperature depression $(\Delta \mathrm{BT})$ is about $4 \mathrm{~K}$. It becomes much larger with increasing particle size. For a particle size of $68.5 \mu \mathrm{m}, \Delta \mathrm{BT}$ is approximately 17 $\mathrm{K}$, for $85.5 \mu \mathrm{m}$ approximately $28 \mathrm{~K}$ and for $128.5 \mu \mathrm{m}$ approximately $52 \mathrm{~K}$.

The plots on the right-hand side show the results at $11.5 \mathrm{~km}$ tangent altitude, which look very different from those at $8 \mathrm{~km}$ tangent altitude. In the window regions about 317 and $322.5 \mathrm{GHz}$, a BT enhancement is observed. This means that more radiation is scattered 

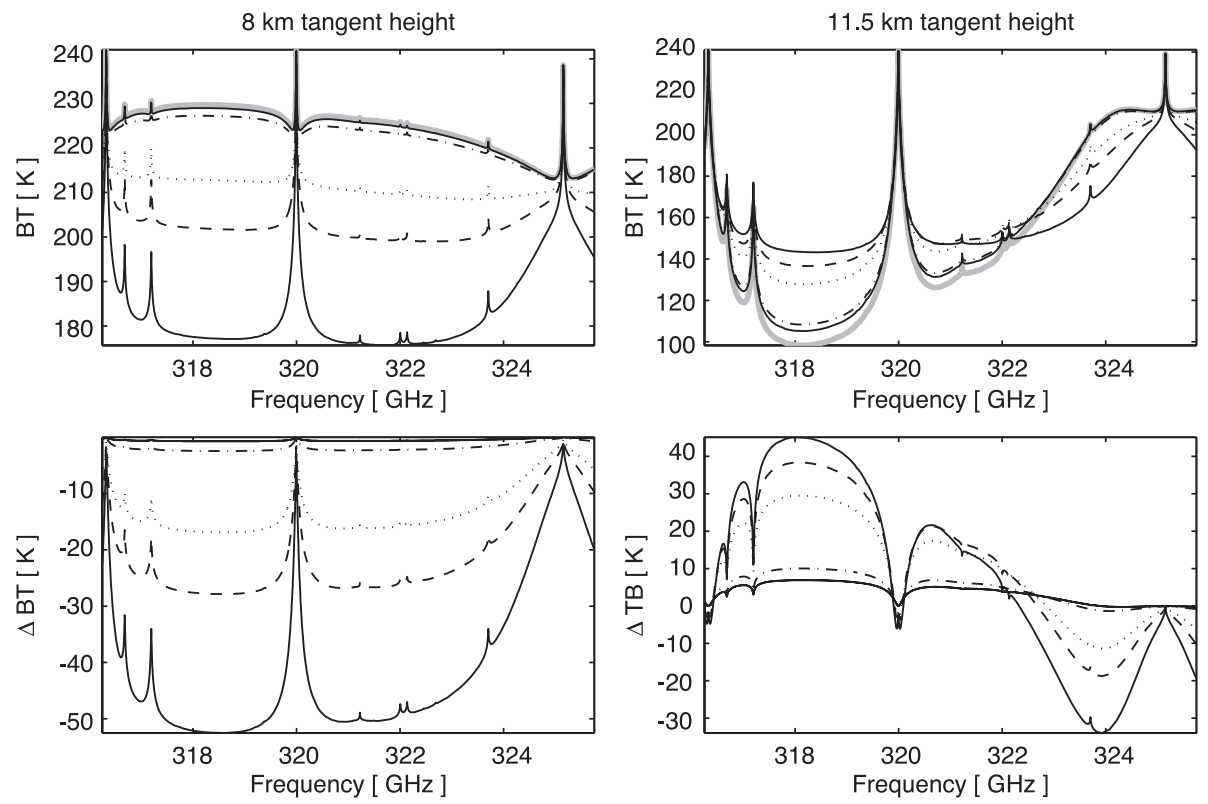

Fig. 3. Impact of particle size. Left panel — limb spectrum at 8-km tangent altitude. Right panel — limb spectrum at 11.5-km tangent altitude. Clear-sky spectrum (grey line), and cloudy spectra for $R_{\text {eff }}=21.5 \mu \mathrm{m}$ (solid), $R_{\mathrm{eff}}=34.0$ $\mu \mathrm{m}$ (dashed-dotted), $R_{\mathrm{eff}}=68.5 \mu \mathrm{m}$ (dotted), $R_{\mathrm{eff}}=85.5 \mu \mathrm{m}$ (dashed) and $R_{\mathrm{eff}}=128.5 \mu \mathrm{m}$ (solid). Top plots show absolute BTs, bottom plots differences from the clear-sky case.

into the line of sight (LOS) than away from the LOS. The largest BT enhancement can be observed at about $318 \mathrm{GHz}$ in the window region of band $\mathrm{C}$. It ranges from about $5 \mathrm{~K}$ for a particle size of $21.5 \mu \mathrm{m}$ and goes up to about $50 \mathrm{~K}$ for a particle size of $128.5 \mu \mathrm{m}$. Above approximately $322.5 \mathrm{GHz}$, we see a BT depression for all particle sizes. The total extinction coefficient consists of particle absorption, particle scattering away from the LOS and gas absorption. If the contribution from the gas absorption is dominant, the scattering effect becomes small. More radiation is absorbed than scattered into the LOS. The BT depression varies from almost $0 \mathrm{~K}$ for the cloud with the smallest particles to approximately $35 \mathrm{~K}$ for the largest particles. The results show that the size of the particles has a very large impact on limb radiances.

\subsection{Impact of cloud altitude}

The impact of cloud altitude on limb radiances is presented in Fig. 4. The IMC is the same as taken for Fig 3. The assumed particle size for those calculations is $34.0 \mu \mathrm{m}$. This is one of the optically thinner clouds. The grey line is the clear-sky spectrum. We see that the cloud at altitude $6-8 \mathrm{~km}$ has a very small impact on the radiances, below $0.5 \mathrm{~K}$ at $318 \mathrm{GHz}$ and even less at higher frequencies. The cloud at $8-10 \mathrm{~km}$ altitude leads to a BT depression of maximal $1.7 \mathrm{~K}$ and the one at $10-12 \mathrm{~km}$ to a BT depression of maximal $2.4 \mathrm{~K}$. 

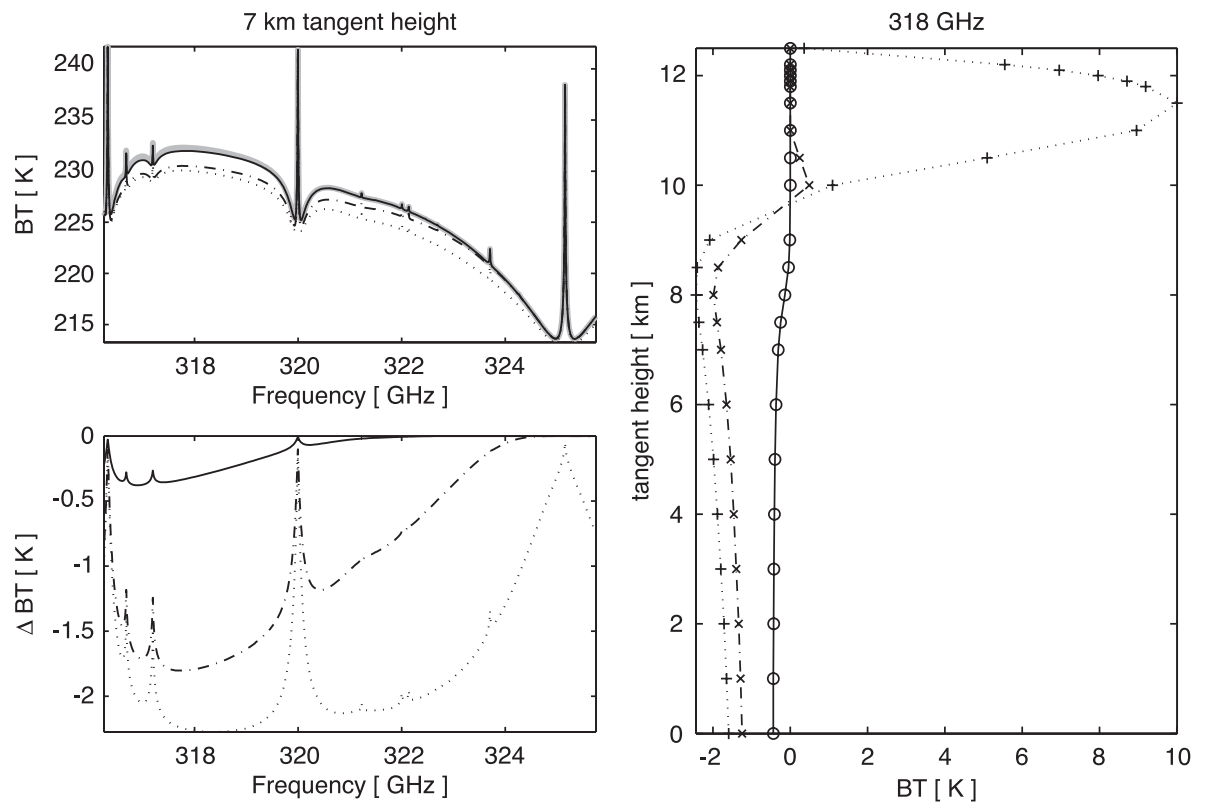

Fig. 4. Impact of cloud altitude. Left panel-limb spectrum at 7-km tangent altitude. Clear-sky spectrum (grey line), and cloudy spectra for cloud altitude 6-8 km (solid), 8-10 km (dash-dotted) and 10-12 km (dotted). Top plot shows absolute BTs, bottom plot differences from the clear-sky case. Right panel: radiances at $318 \mathrm{GHz}$ as a function of cloud altitude; 6-8 km (solid), 8-10 km (dash-dotted) and 10-12 km (dotted).

On the right side, the BT is plotted as a function of tangent altitude. The lowest cloud shows in all tangent altitudes only a very small impact. The cloud at $8-10 \mathrm{~km}$ altitude leads to a BT depression at tangent altitudes up to $9 \mathrm{~km}$ and then to small BT enhancement up to $10.5-\mathrm{km}$ tangent altitude. The cloud signal is observed up to $10.5 \mathrm{~km}$ as the scattering properties are interpolated linearly between the grid points. As mentioned above, the cloud ranges from 7.5 to $10.5 \mathrm{~km}$ in this case. For the highest cloud, we also observe BT depression up to $9 \mathrm{~km}$ tangent altitude and very high BT enhancement from 10 to $12 \mathrm{~km}$ tangent altitude. The enormously higher BT enhancement for the high cloud is due to the fact that in lower altitudes, water vapor absorption is very large.

\subsection{Impact of particle shape}

The results of limb spectra for clouds consisting of cylindrical particles with different aspect ratios varying from 0.3 to 4.0 is shown in Fig. 5. The calculations were done for the same IMC as taken in the previous calculations, but here, a larger mean particle size of $68.5 \mu \mathrm{m}$ has been taken. We can see immediately that the difference between the curves is small compared to the scattering effect itself. At $8 \mathrm{~km}$ tangent altitude, the total BT depression is about $17 \mathrm{~K}$. The difference between the spectra for different aspect ratios is only approximately $2 \mathrm{~K}$. At $11.5 \mathrm{~km}$ tangent altitude, the maximal BT enhancement is about $30 \mathrm{~K}$ and the differences for different shapes are again in the order of $2 \mathrm{~K}$. We have 

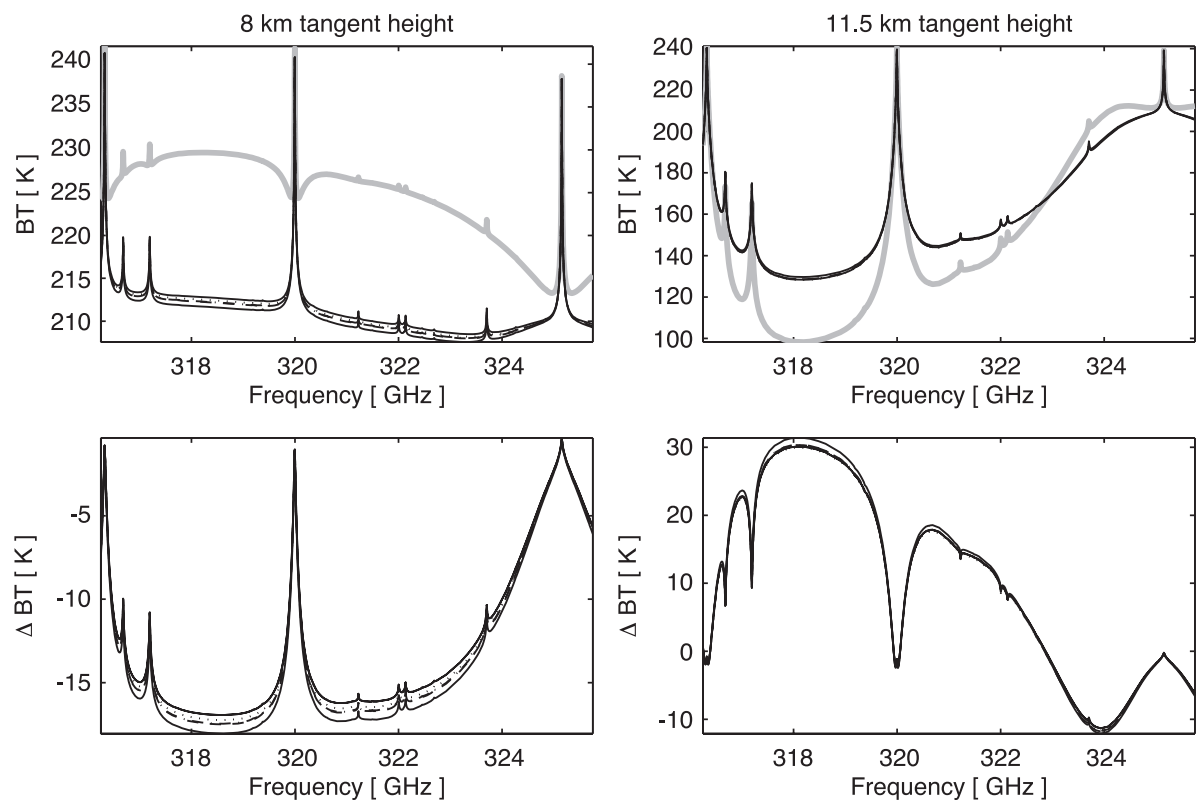

Fig. 5. Impact of particle shape. Left panel—limb spectrum at 8-km tangent altitude. Right panel—limb spectrum at $11.5-\mathrm{km}$ tangent altitude. Clear-sky spectrum (grey line), and cloudy spectra for aspect ratios 0.3 (solid), 0.5 (dashed-dotted), 1.0 (dotted), 2.0 (dashed) and 4.0 (solid). Top plots show absolute BTs, bottom plots differences from the clear-sky case.

not studied in detail the effect of particle shape yet. At this point, it does not make sense to interpret the results in detail, for example, whether plates (aspect ratio $<1$ ) show a higher signal than cylinders (aspect ratio $>1$ ). We may only conclude that the effect of particle shape is much smaller than the effects of particle size and cloud altitude.

\subsection{Cloud scenarios with correlation between IMC and $R_{\text {eff }}$}

Results of the calculations for the "realistic" clouds are shown in Fig. 6. The left side shows the results for $8 \mathrm{~km}$ tangent altitude. The impact of the optical thinnest cloud $\left(\mathrm{IMC}=4 \times 10^{-5} \mathrm{~g} / \mathrm{m}^{3}, R_{\text {eff }}=21.5 \mu \mathrm{m}\right)$ is very small; the spectrum cannot be distinguished from the clear-sky spectrum. The impact of the second cloud $\left(\mathrm{IMC}=1.6 \times 10^{-3} \mathrm{~g} / \mathrm{m}^{3}\right.$, $R_{\text {eff }}=34.0 \mu \mathrm{m}$ ), which is the one used for studying the effect of cloud altitude, is very small compared to the other cloud scenarios. For the optically thickest cloud, the BT depression may go up to $120 \mathrm{~K}$. The highest BT enhancement at $11.5 \mathrm{~km}$ is observed for a medium cloud thickness $\left(\mathrm{IMC}=8 \times 10^{-3} \mathrm{~g} / \mathrm{m}^{3}, R_{\mathrm{eff}}=68.5 \mu \mathrm{m}\right)$. The reason is that in case of very thick clouds, mainly radiation scattered into the LOS in the upper part of the cloud contributes to the spectra. It is very probable that radiation scattered into the LOS in the lower or middle part of the cloud is again absorbed or scattered away from the LOS by a cloud particle. For thinner clouds, most radiation, which is scattered once into the LOS, will continue to propagate into this direction without being disturbed by other cloud particles. 

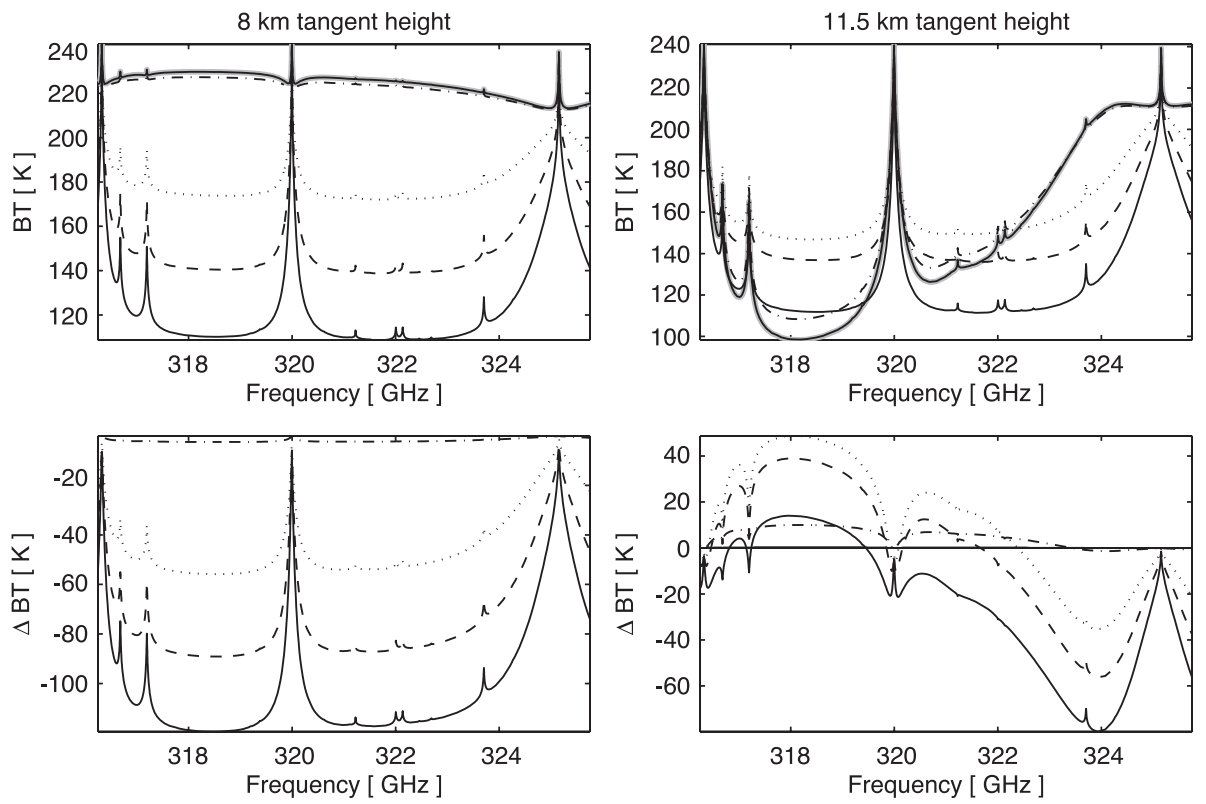

Fig. 6. Impact of IMC. Left panel—limb spectrum at 8-km tangent altitude. Right panel—limb spectrum at $11.5-\mathrm{km}$ tangent altitude. Clear-sky spectrum (grey line), and cloudy spectra for IMC $=4 \times 10^{-5}$ (solid), $1.6 \times 10^{-3}$ (dashed-dotted), $8 \times 10^{-3}$ (dotted), 0.16 (dashed) and 0.04 (solid) and the corresponding particle sizes. Top plots show absolute BTs, bottom plots differences from the clear-sky case.

Although the microphysical cloud properties, i.e., particle size and IMC, are "realistic", a limb instrument would observe smaller scattering signals. As mentioned above, these calculations can only be taken as an upper limit of scattering effect because we have taken the 1D mode of the model which corresponds to a homogeneous cloud cover. This assumption is unrealistic, particularly for the intense cloud cases, which correspond to clouds of limited horizontal extent.

\subsection{Spectra for different frequency bands}

In Fig. 7 results for MASTER bands $\mathrm{B}$ and $\mathrm{C}$ are presented. The spectra are shown for the tangent altitudes $3,9,10.5$ and $11.5 \mathrm{~km}$. The top panels show the clear-sky spectra, the middle panels the spectra in presence of cloud and the bottom panels show the difference between cloudy and clear-sky spectra $\Delta \mathrm{BT}$. IMC, $R_{\text {eff }}$ and cloud altitude are defined in Table 1.

In band B, there is an oxygen line at approximately $298.5 \mathrm{GHz}$. The other visible spectral lines are due to ozone. For 3-km tangent altitude, there is a BT depression of maximal $1.5 \mathrm{~K}$. All other tangent altitudes show a BT enhancement due to clouds. The difference between cloudy and clear-sky spectrum is very small $(<3 \mathrm{~K})$ for tangent altitude $9 \mathrm{~km}$. This tangent altitude is below the cloud. As the considered cloud is rather optically thin, we can already see at $9 \mathrm{~km}$ a BT enhancement. For $10.5 \mathrm{~km}$ tangent 

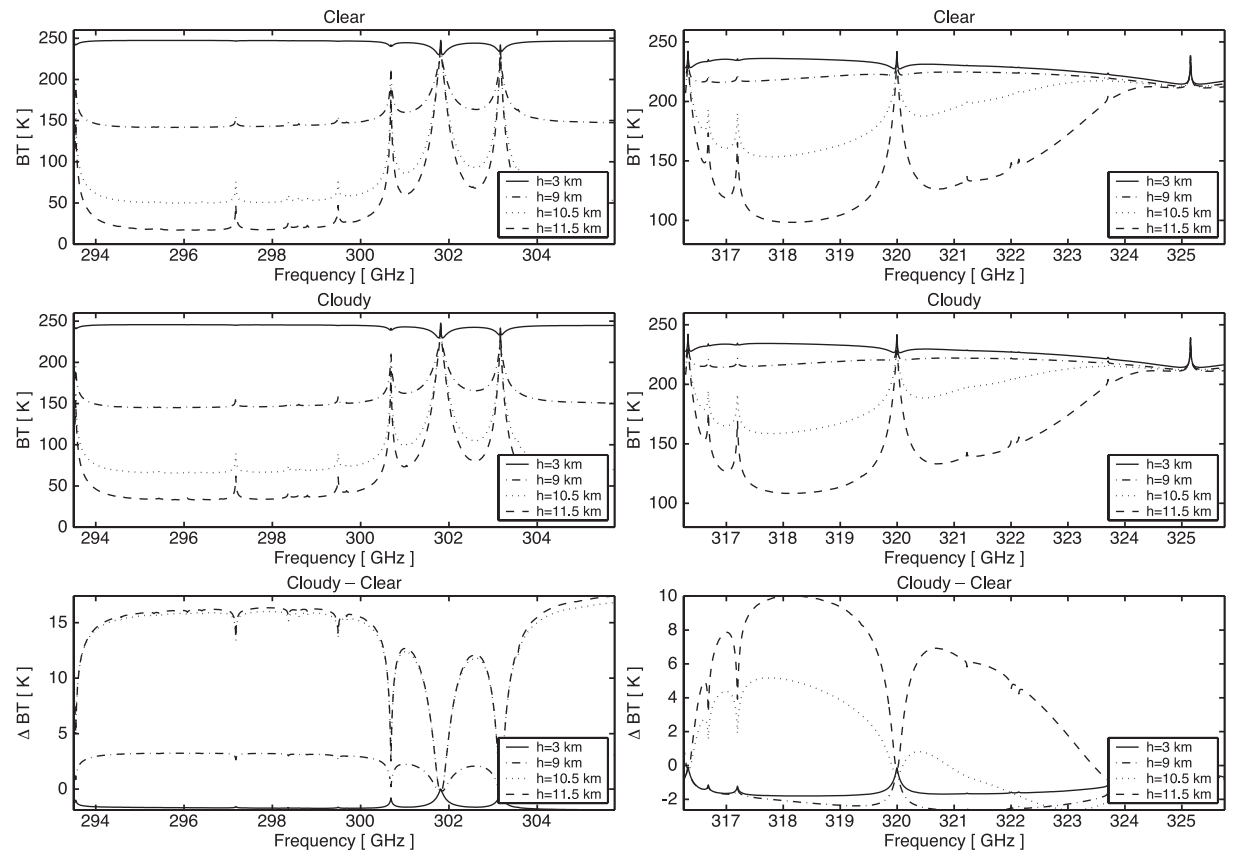

Fig. 7. Limb spectra at different tangent altitudes. Left panel: band B (294-304 GHz). Right panel: band C (317$326 \mathrm{GHz})$.

altitude, the enhancement becomes larger, the maximum can be found at approximately $294.5 \mathrm{GHz}$ and it is about $7 \mathrm{~K}$. Even higher enhancement of about $8.5 \mathrm{~K}$ is observed at $11.5 \mathrm{~km}$ tangent altitude.

In band $\mathrm{C}$, there are ozone lines around 317 and $320 \mathrm{GHz}$. The line at approximately $325 \mathrm{GHz}$ is a water vapor line with a high gas absorption. This means that the largest scattering effect can be observed in the window regions around the ozone line at 320 GHz. Like in band B, we see a BT enhancement for the tangent altitudes inside the cloud $(10.5$ and $11.5 \mathrm{~km})$. The maximum at $11.5 \mathrm{~km}$ at about $318 \mathrm{GHz}$ is with $10 \mathrm{~K}$ brightness temperature difference larger than the maximum in band $\mathrm{B}$, although the total gas absorption in band $\mathrm{C}$ is higher than in band $\mathrm{B}$. The explanation are the single scattering properties. The scattering coefficient increases with frequency in the microwave range (cf. Fig. 2). At $11.5 \mathrm{~km}$ tangent altitude (upper part of the cloud), most radiation is scattered into the LOS. At $10.5 \mathrm{~km}$ tangent altitude, $\Delta \mathrm{BT}$ is smaller compared to band $\mathrm{B}$. As the scattering coefficient is larger in band $\mathrm{C}$, the probability for multiple scattering is increased. If radiation is scattered into the LOS at $10.5 \mathrm{~km}$, there is a large probability that it is again scattered out of the LOS during the propagation from 10.5 to $12 \mathrm{~km}$. The spectrum at tangent altitude $9 \mathrm{~km}$ shows already a BT depression in band C; the amount is less than $2 \mathrm{~K}$. The BT depression for $3 \mathrm{~km}$ tangent altitude is maximal $2.5 \mathrm{~K}$, about $1 \mathrm{~K}$ larger than the maximal depression in band B. This is the expected result, as the extinction coefficient is increased for higher frequencies. 
Fig. 8 shows the results for bands D and E. In band D, there are ozone lines around $343.3 \mathrm{GHz}$ and an oxygen line at approximately $345.3 \mathrm{GHz}$. The total gas absorption is similar to band B. As the scattering coefficient is larger for higher frequencies, the BT enhancement from the cloud at 10.5 - and $11.5 \mathrm{~km}$ tangent altitude is increased; it is, in this case, maximal 11 and $14 \mathrm{~K}$, respectively. At $9 \mathrm{~km}$ tangent altitude, the cloud effect is very small; only depression of less than $1 \mathrm{~K}$ is observed. The BT depression at $3 \mathrm{~km}$ tangent altitude is approximately $3 \mathrm{~K}$.

Band $\mathrm{E}$ is in a much higher frequency region with much larger scattering coefficients than the other bands. But also the total gas absorption is the largest in this band. Only for a tangent altitude of $11.5 \mathrm{~km}$ we see a BT enhancement throughout the whole band; the maximum value is about $17 \mathrm{~K}$. The smallest brightness temperature difference in band $\mathrm{E}$ is observed for $10.5 \mathrm{~km}$ tangent altitude, which corresponds to an altitude inside the cloud. At $9 \mathrm{~km}$ tangent altitude, the BT depression is larger (max. $12 \mathrm{~K}$ ) than at $3-\mathrm{km}$ tangent altitude $(\max .8 \mathrm{~K})$. As the propagation path through the cloud is longer for a limb scan with 9-km tangent altitude than for a scan with $3 \mathrm{~km}$ tangent altitude and the cloud extinction is large, more BT depression is observed at a tangent altitude of $9 \mathrm{~km}$.

\subsection{Comparison with nadir radiances}

Compared to nadir radiances, limb radiances are more complex. In nadir geometry, clouds always lead to a brightness temperature depression compared to the clear-sky
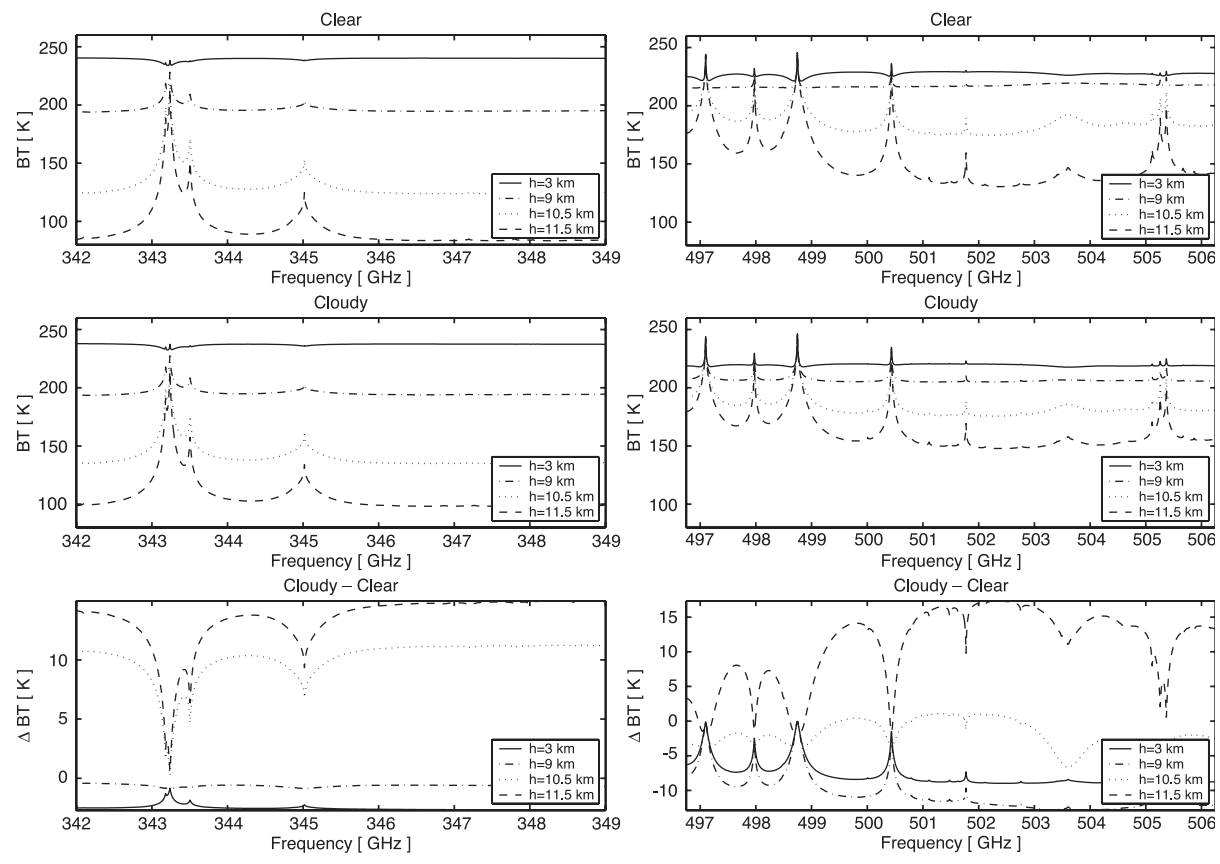

Fig. 8. Limb spectra at different tangent altitudes. Left panel: band D (342-349 GHz). Right panel: band E $(496-506 \mathrm{GHz})$. 
radiances. Nadir instruments look at the tropopause and the ground, where the major source of microwave radiation is located. Clouds absorb and scatter part of the radiation out of the LOS. When we consider the lower atmosphere and the emitting ground as major sources of radiation, the propagation of radiation is upwelling. In nadir geometry, we measure the upwelling radiation; there cannot be an enhancement in this direction due to scattering according to the law of energy conservation.

In limb geometry, clouds usually lead to brightness temperature depression if the tangent altitude lies below the cloud. But it can also lead to a BT enhancement if the tangent altitude is inside the cloud because part of the upwelling radiation from the earth's surface and the lower atmosphere is scattered into the LOS. In the clear-sky case, the sensor does not see thermal emission from the lower atmosphere at all.

The impact of cloud in nadir geometry is smaller, as the path length through the cloud is much shorter. Of course, the path length through the cloud for a limb measurement depends on the horizontal extent of the cloud. As clouds have an infinite extent in a 1D model, the radiances are overestimated for most cases, as cloud coverage is normally horizontally inhomogeneous. But still, the path length through the clouds is larger in limb. The clouds presented in this study are mostly optically thin. The highest brightness temperature depression in nadir observing geometry by the clouds used to study the impact of particle size, case (1), is only $1.7 \mathrm{~K}$. Only case (4),
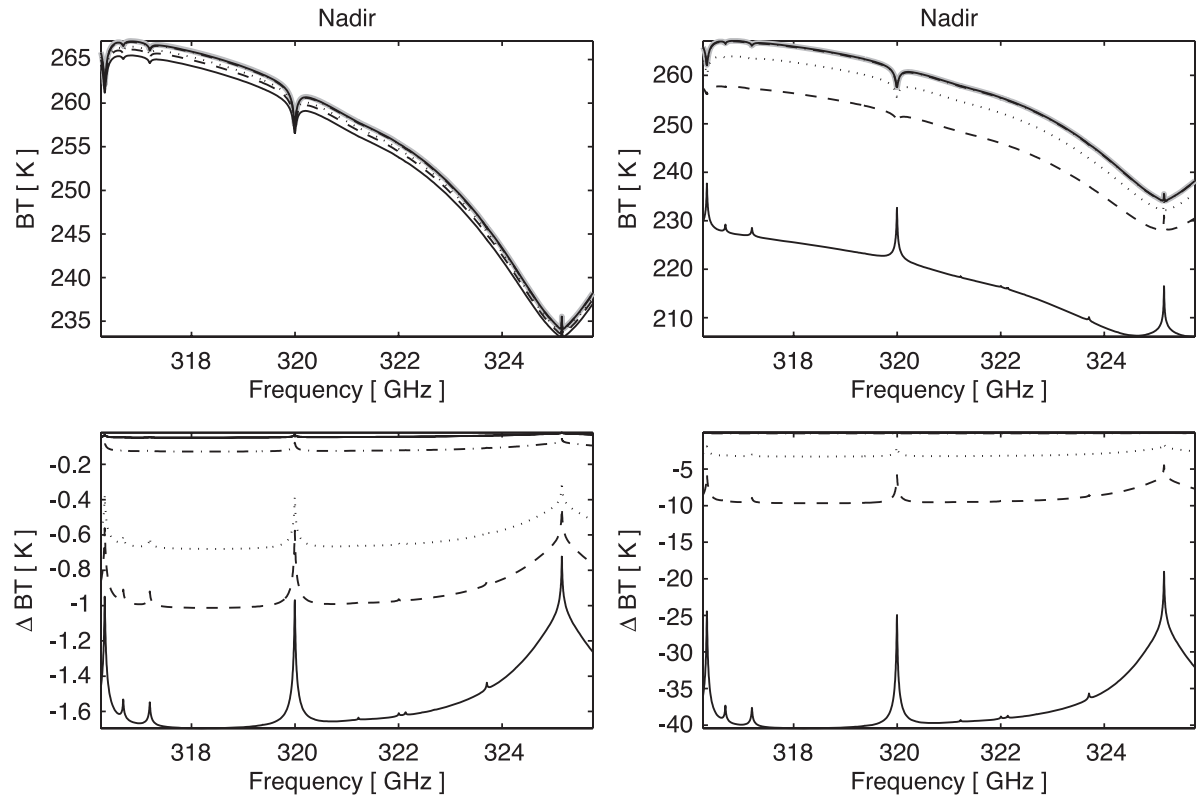

Fig. 9. Effect of clouds in nadir geometry. Left panel—case (1): clear-sky spectrum (thick line), and cloudy spectra for $R_{\mathrm{eff}}=21.5 \mu \mathrm{m}$ (solid), $R_{\mathrm{eff}}=34.0 \mu \mathrm{m}$ (dashed-dotted), $R_{\mathrm{eff}}=68.5 \mu \mathrm{m}$ (dotted), $R_{\mathrm{eff}}=85.5 \mu \mathrm{m}$ (dashed) and $R_{\text {eff }}=128.5 \mu \mathrm{m}$ (solid). Right panel—case (4): clear-sky spectrum (thick line), and cloudy spectra for $\mathrm{IMC}=4 \times 10^{-5}$ (solid), $1.6 \times 10^{-3}$ (dashed-dotted), $8 \times 10^{-3}$ (dotted), 0.16 (dashed) and 0.04 (solid) and the corresponding particle sizes. Top plots show absolute BTs, bottom plots differences from the clear-sky case. 
where we increased particle size and IMC simultaneously, shows higher BT depression (see Fig. 9).

\section{Conclusions and outlook}

The results (Figs. 3 and 6) show that the ice mass content and the size of the cloud particles are both important. Consider the dotted line in Fig. 3, corresponding to an IMC of $1.6 \times 10^{-3} \mathrm{~g} / \mathrm{m}^{3}$ and an effective particle radius of $64.0 \mu \mathrm{m}$, and the dotted line in Fig. 6, corresponding to the same particle size but a five times higher IMC $\left(8 \times 10^{-3} \mathrm{~g} / \mathrm{m}^{3}\right)$. The maximum BT depression at $8 \mathrm{~km}$ tangent altitude is increased by a factor of 2.5 when we compare the two results. When the effective particle size is doubled from 34.0 to $68.5 \mu \mathrm{m}$, the BT depression is enhanced by a factor of 9. To estimate in detail the effect of particle size and IMC, a sensitivity study for more sizes and IMC is necessary. The strong impact of both particle size and IMC shows that in cloud retrievals, it will be a challenge to obtain IMC and particle size simultaneously, as they both lead to an enhancement of the scattering effect. For retrievals with clouds, further investigations are required.

The highest effect of cloud scattering can be observed in the microwave window regions of the spectral bands. The total extinction coefficient consists of gaseous extinction and particle extinction. When the gaseous extinction cross section dominates the total extinction, the scattering effect becomes smaller. In the line centers, there is no difference between cloudy and clear-sky spectrum. At the center frequencies, the water vapor path is so high that the transmission from the cloud to the sensor is zero; thus, the existence of the cloud does not affect the measured radiance. As gas absorption is very high at low altitudes, the cloud altitude has a big effect on the scattering signal. At low altitudes, gas absorption is dominant; therefore, the scattering effect for low clouds is very small. Cirrus clouds can exist in altitudes above $10 \mathrm{~km}$. Here, gas absorption is low, so the scattering signal can be very large. The scattering coefficients increase with frequency. But the scattering effect does not necessarily increase with frequency. It also depends on the gas absorption characteristics of the considered frequency region.

The particle shape is less important than the particle size and the IMC, at least for the cloud particles studied here. For higher aspect ratios and more asymmetrical particles, the impact is most probably larger. For asymmetrical particles, the radiation will be polarized due to scattering. Using ARTS, the polarization effect can be investigated in future studies.

\section{Acknowledgements}

We thank Michael Mishchenko and Stephan Warren for making available the T-matrix program and the refractive index program, respectively. Furthermore, we thank Oliver Lemke for programming assistance and technical support.

This work was funded by the German Federal Ministry of Education and Research (BMBF), within the AFO2000 project UTH-MOS, grant 07ATC04, and the DLR project 
SMILES, grant 50EE9815. It was co-funded by the ESA study UTLS, ESTEC Contract No $15457 / 02 / \mathrm{NL} / \mathrm{MW}$. It also is a contribution to COST Action 723 'Data Exploitation and Modeling for the Upper Troposphere and Lower Stratosphere'.

\section{References}

Anderson, G.P., Clough, S.A., Kneizys, F.X., Chetwynd, J.H., Shettle, E.P., 1986. AFGL atmospheric constituent profiles $(0-120 \mathrm{~km})$. Technical Report TR-86-0110, AFGL.

Arking, A., 1991. The radiative effects of clouds and their impact on climate. Bull. Am. Meteorol. Soc. 72, $795-813$.

Bohren, C., Huffman, D.R., 1998. Absorption and Scattering of Light by Small Particles, Wiley Science Paperback Series, New York, NY.

Buehler, S.A., 1999. Microwave Limb Sounding of the Stratosphere and Upper Troposphere. Berichte aus der Physik. Shaker Verlag GmbH, PO-Box 1290, D 52013 Aachen. PhD thesis, University of Bremen, ISBN 3-8265-4745-4

Buehler, S.A., Eriksson, P., Kuhn, T., von Engeln, A., Verdes, C., 2003. ARTS, the atmospheric radiative transfer simulator. J. Quant. Spectrosc. Radiat. Transfer (in press).

Donovan, D.P., 2003. Ice-cloud effective particle size parametrization based on combined lidar, radar reflectivity, and mean Doppler velocity measurements. J. Geophys. Res. 108 (D18), 4573 (doi:10.1029/2002JD003469).

Eriksson, P., Buehler, S.A., Emde, C., Sreerekha, T.R., Melsheimer, C., Lemke, O. ARTS User Guide, University of Bremen, 2003. Regularly updated versions available at www.sat.uni-bremen.de/arts.

Evans, K.F., Walter, S.J., Heymsfield, A.J., Deeter, M.N., 1998. Modeling of submillimeter passive remote sensing of cirrus clouds. J. Appl. Meteorol. 37, 184-205.

Evans, K.F., Walter, S.J., Heymsfield, A.J., McFarquhar, G.M., 2002. Submillimeter-wave cloud ice radiometer: simulations of retrieval algorithm performance. J. Geophys. Res. 107 (D3), ACC-2-1-ACC-2-21 (10.1029/ 2001JD000709).

Hoepfner, M., Emde, C., 2003. Comparison of single and multiple scattering approaches for the simulation of limb-emission observations in the mid-IR. J. Quant. Spectrosc. Radiat. Transfer (in press).

Kerridge, B., Jay, V., Reburn, J., Siddans, R., Latter, B., Dudia, A., Lama, F., Hoepfner, M., Steck, T., Stiller, G., von Clarmann, T., Grabowski, U., Buehler, S., Miao, J., Emde, C., Murtagh, D., Eriksson, P., Baran, A., Wickett, M., 2003. Consideration of mission studying chemistry of the UTLS, progress report 6, Technical report, ESTEC, November.

Kinne, S., et al., 1997. Cirrus cloud radiative and microphysical properties from ground observations and in situ measurements during FIRE 1991 and their applications to exhibit problems in cirrus solar radiative transfer modeling. J. Atmos. Sci. 54, 2320-2344.

Mishchenko, M.I., Travis, L.D., 1998. Capabilities and limitations of a current FORTRAN implementation of the T-matrix method for randomly oriented rotationally symmetric scatterers. J. Quant. Spectrosc. Radiat. Transfer 60, 309-324

Mishchenko, M.I., Travis, L.D., Lacis, A.A., 2002. Scattering, Absorption, and Emission of Light by Small Particles, Cambridge University Press, Cambridge.

Read, W.G., Waters, J.W., Wu, D.L., Stone, E.M., Shippony, Z., Smedley, A.C., Smallcomb, C.C., Oltmans, S., Kley, D., Smit, H.G.J., Mergenthaler, J.L., Karki, M.-K., 2001. UARS microwave limb sounder upper tropospheric humidity measurement: Method and validation. J. Geophys. Res. 106, 32207-32258.

Rothman, L.S., Rinsland, C.P., Goldman, A., Massie, S.T., Edwards, D.P., Flaud, J.-M., Perrin, A., CamyPeyret, C., Dana, V., Mandin, J.-Y., Schroeder, J., McCann, A., Gamache, R.R., Wattson, R.B., Yoshino, K., Chance, K.V., Jucks, K.W., Brown, L.R., Nemtchinov, V., Varanasi, P., 1998. The HITRAN molecular spectroscopic database and HAWKS (HITRAN atmospheric workstation): 1996 edition. J. Quant. Spectrosc. Radiant. Transfer 60, 665-710.

Skofronik-Jackson, G.M., Gasiewski, A.J., Wang, J.R., 2002. Influence of microphysical cloud parametrizations on microwave brightness temperatures. IEEE Trans. Geosci. Remote Sens. 40, 1.

Sreerekha, T.R., Buehler, S.A., Emde, C., 2002. A simple new radiative transfer model for simulating the effect of cirrus clouds in the microwave spectral region. J. Quant. Spectrosc. Radiant. Transfer 75, 611-624. 
Stubenrauch, C.J., Holz, R., Chedin, A., Mitchell, D.L., Baran, A.J., 1999. Retrieval of cirrus ice crystal sizes from 8.3 and $11.1 \mathrm{~mm}$ emissivities determined by the improbed initialization inversion of TIROS-N operational vertical sounder observations. J. Geophys. Res. 104 (D24), 31793-31808.

Wang, P.-H., Minnis, P., McCormick, M.P., Kent, G.S., Skeens, K.M., 1996. A 6-year climatology of cloud occurrence frequency from stratospheric aerosol and gas experiment II observations (1985-1990). J. Geophys. Res. 101 (29), 407-429.

Warren, S., 1984. Optical constants of ice from the ultraviolet to the microwave. Appl. Opt. 23, 1206-1225.

Wu, D.L., Read, W.G., Dessler, A.E., Sherwood, S.C., Waters, J.W., 2003. UARS MLS cloud ice measurements and implications for $\mathrm{H}_{2} \mathrm{O}$ transport near the tropopause. J. Atmos. Sci. (submitted for publication). 